\title{
Prevalence of Out-of-Hospital Sudden Cardiac Death in Moscow in 2005-2009
}

\author{
Leonid Makarov, ${ }^{1}$ Vera Komoliatova, ${ }^{1}$ Natalia Fedina, ${ }^{1}$ and Yuri Solokhin ${ }^{2}$ \\ ${ }^{1}$ Center for Syncope and Cardiac Arrhythmias, Russian Federal Medico Biology Agency, \\ Central Children Clinical Hospital FMBA of Russia, 20 Moskvorechie Street, 115409 Moscow, Russia \\ ${ }^{2} 2$ nd Thanatology Department of Forensic Medical Examination Bureau of the Health Care Department of Moscow, \\ 3/2 Tarny proezd, 115516 Moscow, Russia
}

Correspondence should be addressed to Leonid Makarov; leonidmakarov@yahoo.com

Received 26 February 2015; Revised 28 April 2015; Accepted 9 May 2015

Academic Editor: Peter N. Lee

Copyright (C) 2015 Leonid Makarov et al. This is an open access article distributed under the Creative Commons Attribution License, which permits unrestricted use, distribution, and reproduction in any medium, provided the original work is properly cited.

Background. The sudden out-of-hospital cardiac death (SOHCD) in Russia is poorly investigated. The aim of study was to determine structure of SOHCD in Moscow. Methods. SOHCD were analyzed according to data for 2005-2009 from the 2nd Thanatology Department of Forensic Medicine of Moscow that serves 2502836 citizens in Moscow. Results. Prevalence of SOHCD was 49.1\% of autopsies for all age groups and in 8.9\% in the group aged 1-45 (22.3 cases per 100000 population/year). The frequency of SOHCD progressively increased with age. Most SOHCD victims (82\%) were males. The diagnosis of cardiomyopathy was prevalent (80$96 \%$ ) in the age 1-45 group; in 11-15 more 30\% had normal heart; after 35 years of age, the role of ischaemic heart disease increased. In $67 \%$ of the people aged 19-25 SOHCD was associated with traces of alcohol (0.3-3.0 promile). Conclusion. The proportion of SOHCD in the Moscow population over all age groups has reached 123.2 per 100000 citizens annually. In the age group 1-45, the prevalence of SOHCD was 22.3 cases per 100000 citizens per year. The risk of SOHCD was greater in males. Possibly the role of alcohol in SOHCD in people older than 20 increased.

\section{Background}

The sudden cardiac death (SCD) is one of the most important problem in medicine [1]. More than $40 \%$ of these patients die suddenly, mostly in an out-of-hospital setting. According to various estimates, SCD occurs in $0.36-1.28$ cases in every 100000 subjects [2-10]. Often, sudden death is the first manifestation of heart disease.

The precise prevalence of SCD in Russia is poorly investigated, especially in young patients, and this precludes evaluation of the problem and determination of possible prophylactic solutions. The aim of this study was to determine the frequency and age distribution of sudden out-of-hospital cardiac death (SOHCD) in young people aged 1-45 in Moscow.

\section{Methods}

The frequency of SOHCD and the structure of its causes in young subjects were analyzed according to data from the 2 nd
Thanatology Department of Forensic Medical Examination Bureau of the Health Care Department of Moscow for 20052009. This bureau serves 29 districts of Moscow and includes records for 2502836 citizens (52.4\% or 1311486 citizens aged $1-45$ years). It also performs forensic medical examination of all bodies of children who suddenly die in out-of-hospital settings in Moscow. From all autopsies, cases of obstruction asphyxia, acute poisoning, ischaemic stroke, and respiratory, renal, hepatic, gastrointestinal, and other disorders were excluded. As a result, the study group was limited to cases of SCD causes, defined in Russian practice as an "acute cardiovascular insufficiency."

Examination of corpses was performed according to Order number 161 of the Russian Ministry of Health of 1/24/2003: "on approval of the instructions on organization and performance of examinations at the forensic medical examination bureau". Each case was accompanied by forensic-chemical blood and urine (renal) investigation for ethyl alcohol in order to evaluate the relationship between 
TABLE 1: Prevalence of out-of-hospital sudden cardiac death in Moscow, 2.5 million Moscow citizens in 2005-2009.

\begin{tabular}{|c|c|c|c|c|}
\hline Years & $\begin{array}{c}\text { SOHCD at age } \\
1-45 \text { years } \\
n \text { (\% of all autopsies) }\end{array}$ & $\begin{array}{l}\text { All autopsies at age } 1-45 \\
\text { years } n \text { (\% of all autopsies) }\end{array}$ & $\begin{array}{l}\text { SOHCD at all ages ( } \% \text { of } \\
31428 \text { autopsies) }\end{array}$ & All autopsies for any reason \\
\hline 2005 & $\begin{array}{c}635 \\
(9.8 \%)\end{array}$ & $\begin{array}{c}2233 \\
(34.3 \%)\end{array}$ & $\begin{array}{c}3080 \\
(47.3 \%)\end{array}$ & 6505 \\
\hline 2006 & $\begin{array}{c}553 \\
(8.5 \%)\end{array}$ & $\begin{array}{c}2227 \\
(34.3 \%)\end{array}$ & $\begin{array}{c}3097 \\
(47.7 \%)\end{array}$ & 6489 \\
\hline 2007 & $\begin{array}{c}558 \\
(8.7 \%)\end{array}$ & $\begin{array}{c}2021 \\
(31.4 \%)\end{array}$ & $\begin{array}{c}3148 \\
(48.9 \%)\end{array}$ & 6432 \\
\hline 2008 & $\begin{array}{c}585 \\
(9.4 \%)\end{array}$ & $\begin{array}{c}1945 \\
(31.2 \%)\end{array}$ & $\begin{array}{c}3193 \\
(51.3 \%)\end{array}$ & 6228 \\
\hline 2009 & $\begin{array}{c}459 \\
(7.9 \%)\end{array}$ & $\begin{array}{c}1706 \\
(29.5 \%)\end{array}$ & $\begin{array}{c}2902 \\
(50.3 \%)\end{array}$ & 5774 \\
\hline ALL & $\begin{array}{c}2790 \\
(8.9 \%)\end{array}$ & $\begin{array}{c}10132 \\
(32.2 \%)\end{array}$ & $\begin{array}{c}15420 \\
(49.1 \%)\end{array}$ & 31428 \\
\hline
\end{tabular}

SOHCD: sudden out-of-hospital cardiac death.

alcohol intoxication and SOHCD. In addition to macroscopic examination of the body organs and tissue morphology at autopsy, in most cases microscopy (histological examination) was performed. As a rule, standard staining with hematoxylin and eosin was performed, with special staining in complex cases-for instance, staining of myocardium by Rego and Van Giesen. "Cardiomyopathy" was diagnosed when several morphological changes were seen-increase in cardiac mass and dilation of cardiac cavities, and an absence of changes in the coronary arteries (sometimes negligible lipoidosis was found, rarely combined with fibrous plaque). Visually, the myocardium in such "cardiomyopathies" was flaccid, with a "cooked meat" or "clay" appearance, sometimes with small foci of cardiosclerosis. Microscopy revealed multiple infiltrates from lymphocytes and lymphohistiocytes, atrophy in combination with hypertrophy of cardiomyocytes with dystrophic changes, fragmentation of cardiomyocytes, and small foci and reticular cardiosclerosis. Frequently, small drops of fatty degeneration of myocardial fibres were noted.

The place of death was registered (as follows: apartment, street, and public place) and also, if was possible, the circumstances of death (previous physical exercise, conflicts, sleep, etc.), data on previous disorders, and data regarding medical anamnesis.

Statistical analysis was performed using the software "Excel" and "Statistica for Windows" version 5.0 (StatSoft, USA).

\section{Results}

The prevalence of SOHCD for the period under analysis is presented in Table 1 . As Table 1 shows, for the study period, SOHCD was responsible, in total, for death in $49.1 \%$ of all autopsies for all age groups combined (123.2 cases per 100000 a year or 3084 SOHCD a year) and in $8.9 \%$ of autopsies in the group aged 1-45 (2790 for 5 years of observation or on average 558 SOHCD a year). When extrapolated to the total population in the area (2502836 citizens), this indicates that the SOHCD frequency for age groups between 1 and 45 years is estimated at 22.3 cases per 100000 population per year. The proportion of SOHCD cases in the $1-45$ age group relative to that of all causes of sudden out-of-hospital death (SOHD) was $18 \%$ (Table 1). Typically there was a relatively constant number of SOHD for each year (varying from 5774 to 6489 over all age groups and from $459-635$ in the group aged 1-45 years).

The percentage of SOHCD for all autopsies progressively increased with age-from $1 \%$ in the age group under 18 years to $44 \%$ in the group aged $41-45$ years. Most ( $82 \%$ ) suddenly deceased people were males. The structure of the pathology in our study was based on the forensic diagnoses in the different age groups presented in Figure 1. The prevalent forensic diagnosis in the analyzed age group was "cardiomyopathy." This diagnosis was predominant in ages from 19 to 35 (8096\%).

In the majority of cases, congenital heart disease was detected in children aged 1-10 years old who died suddenly; after 35 years of age, the role of ischaemic heart diseasesmyocardial infarction, acute coronary insufficiency, postinfarction cardiosclerosis, and atherosclerosis combined with cardiosclerosis-increased. Arterial hypertension and other diseases accounted for $7 \%$ and $1 \%$ of forensic diagnoses, respectively.

In people 20-45 years old, sudden death was registered mainly at home. For those aged below 20 years, $50 \%$ of cases death occurred outdoors, often during physical exercise. The data on the circumstances of death and some of the forensic diagnoses were available in 54 cases subjects 120 years (Table 2 ). An especially high percentage of alcohol intoxication was found in young people of aged 19-25 (67\%). The amount of alcohol in blood tests was estimated in the range from $0.3-0.5$ per $\mathrm{mL}$. (insignificant influence of alcohol or weak alcohol intoxication) to $2.5-3.0$ per $\mathrm{mL}$. (strong alcohol intoxication). If the alcohol concentration was higher, the cause of death was termed "toxic action of ethyl alcohol," and these cases were excluded from our analysis in spite of 


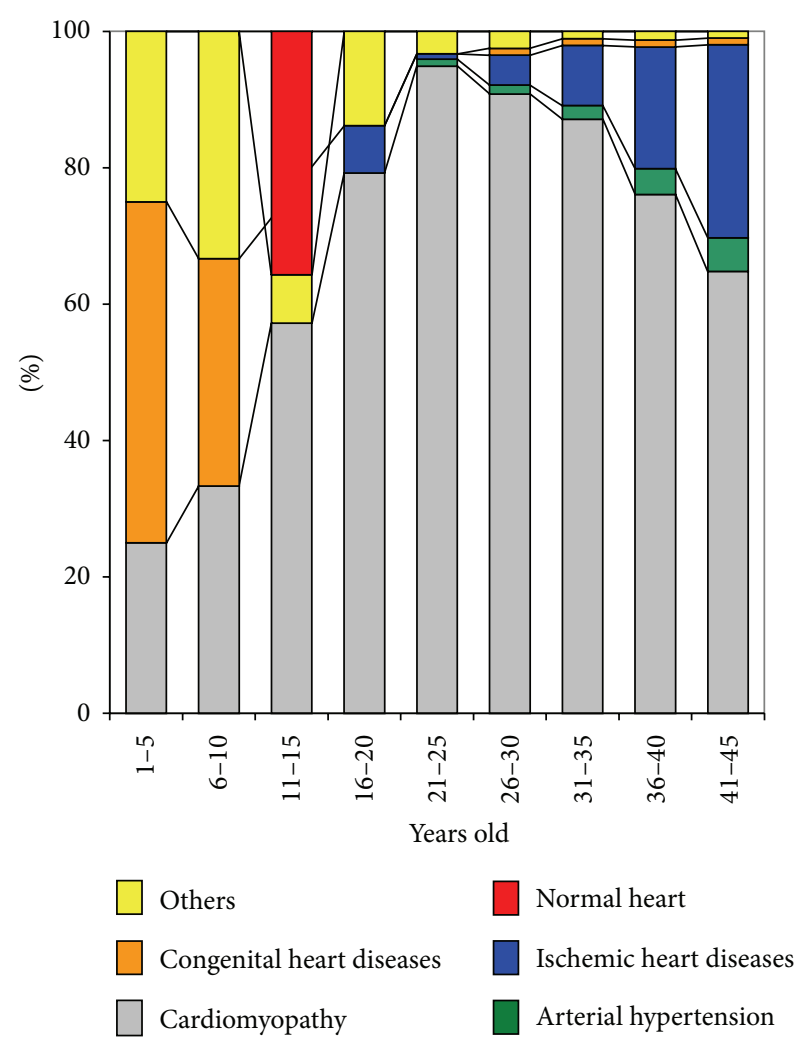

FIGURE 1: Forensic diagnosis in out-of-hospital cardiac death in 2790 people aged 1-45 years in Moscow in 2005-2009.

TABLE 2: Place and age of out-of-hospital cardiac death in 54 people aged 1-20 years in Moscow in 2005-2009.

\begin{tabular}{lcccc}
\hline Place (\%)/age & $1-5$ & $6-10$ & $11-15$ & $16-20$ \\
\hline Home & $6(75 \%)$ & $1(33 \%)$ & $6(43 \%)$ & $14(48 \%)$ \\
Street & & & $3(21 \%)$ & $9(31 \%)$ \\
Near home & & & $2(14 \%)$ & \\
Public place and others & $2(25 \%)$ & $2(67 \%)$ & $3(21 \%)$ & $6(21 \%)$ \\
TOTAL & 8 & 3 & 14 & 29 \\
\hline
\end{tabular}

autopsy evidence of cardiovascular changes. If a history of prolonged alcohol consumption or drug usage was evident and if some evidence of prolonged exogenous intoxication was noted (liver or brain changes), "alcohol cardiomyopathy" or "toxic cardiomyopathy" was diagnosed.

\section{Discussion}

According to the common definition, SCD is the process of death, related to cardiac causes, preceded by sudden loss of consciousness within 1 hour after the appearance of symptoms; the patient can suffer from heart diseases, but the time and mechanism of death are unpredictable [1]. As sometimes it is impossible to precisely determine timing from the development of first symptoms to an out-of-hospital death, the "1 hour" criterion of SOHCD is often widened to include all cases of death from 6-24 h after development of the first symptoms [1]. In forensic medicine, usage of the named criterion is difficult, as the forensic expert in most cases has no data on premortal circumstances, including the time of the beginning of a fatal attack.

In Russian forensic medicine "sudden death" is a prevalent term, although clinicians prefer the term "unexpected death." The concept of "sudden death" in forensic medicine has remained largely unchanged during the last 150 years. In 1856 the well-known Russian forensic pathologist Chistovich defined "sudden death" as follows: "as the law and logic say, only those who were completely healthy and die suddenly from unexpected cause can be accounted for as suddenly dead."

In forensic examination of the bodies of people who suddenly or unexpectedly die outside of health facilities, a forensic expert must exclude all possible causes of rapid death (including violent death). Among these causes are diseases, acute poisoning, and some types of mechanical asphyxia. Therefore, the Russian Health Ministry issued Order number 161 of 24.01.03, "On approval of the instructions on organization and performance of expert examinations at the forensic medical examination bureau" (according to which the autopsies in our study were performed), wich is regulation the opening of three body cavities (the skull, thorax, and abdomen) and thorough examination (dissection) of all organs and tissues. In addition to morphometry and visual examination of body organs and tissues, forensic examination of the body supposes several additional laboratory investigations-chemical, histological, bacteriological, virological, and so forth.

The prevalence of SOHCD is actively studied all over the world. Chugh et al. [8] analyzed SOHCD cases in one district in Oregon, USA (a total of 660486 citizens) [8] and measured the incidence of SOHCD as 53 cases per 100000 population per year (the mean age of the deceased was 69), which was $5.6 \%$ of all deaths. In 10 regions of the USA and Canada (with a total population of 21.4 million), in 2006-2007 a total of 20520 cases of cardiac arrest were registered in an outof-hospital setting $(0.1 \%$ of the population or 95.9 cases per 100000 population) [6]. In our study, the total percentage of people dying suddenly from all causes in all age groups was higher $-8.9 \%$ of all autopsies - which is comparable with data from other studies in countries with higher socioeconomic and health care status. In the study of Morentin et al. [9], sudden death accounted for $9 \%$ of all causes of death in people who died aged 1-19 (1.7 cases per 100000 population a year). In $2.6 \%$ of analysed cases, death had an obvious cardiac genesis and in $2.5 \%$ of cases was unexplained, possibly arrhythmogenic; in total, these causes accounted for 5.5\% of all causes of death. In a study of the structure and frequency of SOHCD in young people aged 12-35 during the last 30 years in Iceland, SOHCD was found to cause $7.7 \%$ of all cases of death or 1.38 cases per 100000 population per year [10]. The retrospectively registered incidence of SOHCD in young people aged 14-35 years in Ireland was 2.85 per 100000 people per year. [11]. 
It is evident from Figure 1 that the only apparently normal hearts were in the 11-20 age group. This is not the finding in most other recent studies in this field $[8,10,12]$. But before 2000 the most authors did not diagnose "normal heart" at the autopsy in children and adolescents. There were first of all "carditis," "cardiomyopathy," "mitral valve prolapse," and other structural heart diseases (observation from 9 articles with 469 cases of SCD in 1-39-year-old subjects during 19791985 in the review of Liberthson [13]). Wren et al. [14] performed assessment of 142 cases of SD during 1985-1994 in northern regions of the UK and revealed only one case of long QT syndrome (LQTS), but in 34\% (!) the reason for SD was epilepsy. Similar data were obtained by Chugh et al. [8] in a study of the hearts of 270 people older than 20 $(42 \pm 14)$ years who experienced sudden death; they found an absence of cardiac and coronary changes in only $5 \%$ of cases (presumably in younger people), compared with the group as a whole $(35 \pm 9)$. But for patients with detected heart pathology (30\%), myocardial changes were typically presentalthough unlikely to be identified as the cause of deathas nonspecific interstitial fibrosis, moderate left ventricular hypertrophy, and mitral valve prolapse [12]. This group is obviously similar to the group of patients with the prevailing forensic diagnosis "cardiomyopathy" in our study; this is further supported by use of almost identical forensic autopsy and pathomorphological examination protocols [12].

In later studies analysis of the nature of causes of SOHCD myocardial pathology was less often the cause of death based on standard forensic exam protocols. The nature of SOHCD causes in young people (12-35 years) in Iceland showed an absence of any changes in the heart and coronary vessels in $17 \%$ of cases [10]. When cases with ante-mortem diagnoses of "cardiac rhythm disorders" (13\%) and "prolonged QT syndrome" (4\%) were added, the total proportion of sudden death among young people without obvious myocardial pathology exceeded one-third of the whole group.

Possibly some forensic diagnoses are often, or even excessively, established in traditional Russian forensic medical practice for young adults, children, and adolescents, as it is difficult to suppose complete absence among causes of SOHCD of diseases such as prolonged or shortened QT interval syndromes, Brugada syndrome, catecholaminergic ventricular tachycardia, other idiopathic life-threatening arrhythmias, and channelopathies. These conditions cannot be identified at forensic autopsy, and the registered minimal (often microscopic) myocardial changes recorded are possibly caused in some cases by the above mentioned diseases against a background of fatal cardiac arrhythmias. This issue could be addressed more precisely by verification of antemortem diagnosis through retrospective analysis of electrocardiograms, clinical picture, family anamnesis, examination of family members, and genetic studies. Such analysis was performed in several studies [15-17]. Tan et al. [16] performed clinical electrocardiographic and molecular genetic evaluation of 43 families with sudden death of young people (aged less than 40 years) without any obvious cardiovascular diseases at autopsy, and in $40 \%$ of cases they found channelopathies in family members: LQTS, Brugada syndrome, catecholaminergic ventricular tachycardia, right ventricle arrhythmogenic dysplasia/cardiomyopathy (ARVD/ARVC), and hypertrophic cardiomyopathy (HCMP). In a similar study, Behr et al. [15] examined 32 families with cases of sudden unexplained death and in $22 \%$ found similar pathologies. Therefore, it seems justified to suggest that SOHCD at a relatively young age, unaccompanied by obvious myocardial and cardiac vascular changes, should indicate examination of the immediate family. van der Werf et al. [17] examined first-degree relatives of young SOHCD victims and found inherited cardiac diseases in 3.6\%. Earlier we described a case of LQTS identified by genetic methods in a 2-year-old girl whose two sisters suddenly died at the age of 4 years [18].

Currently in Russia there are problems in the formulation of diagnoses, the assessment of autopsy data in relation to the protocol and its implementation in various settings, and algorithms for the evaluation of changes found. These differences currently cause substantial dissociation between clinical and forensic diagnoses and complicate detection of the cause of SOHCD and development of preventative approaches. Our study revealed that minimum changes in the heart often arising as fatal arrhythmias could be diagnosed as "cardiomyopathy of not clear etiology," even in the presence at the patient of lifetime diagnosis, for example, of long QT or Brugada syndromes. Only the collaboration of clinical physicians and forensic in the present study allowed for the first time in Russia forensic physicians to become conscious that an autopsy can miss the changes which are the reason for sudden death. But so far they reached the diagnosis only in group of children of school age, though personally they recognize that absence of these patients in the other age groups (as well as in all modern researches in the world) is illogical.

This issue remains unresolved in other countries as well in some other countries. Comparing data on SOHCD causes in young athletes from two internationally renowned schools, Corrado et al. [19] and Maron et al. [20] found that in Italy the main cause of SOHCD in young athletes is ARVD/ARVC (24\% of cases) [19], but in the USA most SOHCD cases are caused by HCMP (36\%) and only $4 \%$ by ARVD [20]. However, when the three main variants of myocardial pathology (ARVD, HCMP, and myocarditis) seen in postmortem examination of young athletes in both countries are combined, their total proportion among all diagnoses is $38 \%$ in Italy [19] and $46 \%$ in the USA [20]. Substantial ethnic or study protocol-related differences seem unlikely, and hence this difference can most probably be related to differing interpretations of similar pathology. Work needs to be carried out on integrating the approaches of different medical schools and specialists through the development of classifications and learning from the cumulative experience of clinicians, pathomorphologists, and forensic medicine specialists throughout the world.

In the present study the majority of sudden deaths were males (82\%). These data are consistent with research by Berdowski et al. [21] where in a population of young victims (younger than 35 years) who had exercise-related SOHCD, males predominated (92\%). In a study by Winkel et al. [22] sudden death also occurred more frequently (63\%) in males 1-18 years. 
SOHCD and cardiac arrest trends have some age-specific features. The frequency of SOHCD in the common population has peaks at 49-70 years [23]. Comparison with data on SOHCD at a younger age is complicated by differences in the grouping of patients by age in the various studies. Einarsson et al. [10] included data on people aged 12 to 35 years who suddenly died in Iceland during 30 years of surveillance. The frequency of out-of-hospital cardiac arrest and of SOHCD in children aged 0-16 in the urban Finnish population (516000 citizens) was found to be 9.8 and 8.9, respectively, per 100000 children [24]. The mean age at the time of death was $2.9 ; 72.2 \%$ of patients died at less than 18 months, and the prevalent cause was sudden infant death syndrome [25]. In our study, children less than 1 year were not included, and sudden infant death syndrome is mostly recorded in this age group. This could explain the lower estimates of SOHCD in children in our youngest study group (1-18 years old).

Most cases of SOHD in people older than 18 years in our study occurred at home; below 18 years the proportions were similar for sudden death at home versus out of home (in public places, often during physical and sport activity) (Table 2). Notably, $94-95 \%$ of sudden death related to physical activities in young people is caused by cardiovascular pathology according to Drezner et al. [25]. In the study by Kuisma et al. [24], sudden cardiac arrest in children and adolescents in public places was closely associated with good prognosis and the restoring of vital parameters in the early start of resuscitation.

In the study by Kida et al. [23], the analysis of 16000 cases of out-of-hospital heart arrest showed survival of $9.8 \%$ in patients younger than 20 years and substantially lower $(0.8 \%)$ in aged patients. As the records of sudden death related to physical exercise in young people in the USA for 20002006 revealed, the rate of survival in the last three years of analysis (2004-2006) virtually doubled compared with that in the earlier years of the study (2000-2003) and reached 14$17 \%$ [25]. In 2006 alone, successful resuscitations following cardiac arrest from use of automated external defibrillators placed in public places had a rate similar to that of electrical defibrillations performed by specialized ambulance teams [25].

One must also note the substantial rate of death associated with detection of alcohol in the patient's blood. This rate of detection was especially high (66.6\%) among young people aged 19-25. The role of alcohol in the pathogenesis of cardiovascular diseases and especially as a risk factor for SCD is not definitely established. On the one hand, protective effects of small doses of alcohol as a cardiovascular risk-lowering factor have been discussed [26], but on the other hand, the role of alcohol in provoking alcoholic cardiomyopathy, lifethreatening arrhythmias in Brugada syndrome [27], atrial fibrillation, and "holiday heart" syndrome [28] is beyond doubt. Alcohol provokes electrical myocardium instability and variability of the QT interval [29], which can favour the development of dangerous ventricular arrhythmias and SOHCD. Thus, there is a need to consider alcohol consumption as an important factor in determining the risk of SOHCD and in the development of measures for its prevention.

\section{Conclusion}

(1) The proportion of SOHCD in the Moscow population over all age groups was 123.2 per 100000 citizens per year. In the age group 1-45 years, the prevalence of SOHCD was 22.3 cases per 100000 citizens per year.

(2) The risk of SOHCD was greater in males. In people 20-45 years old, sudden death was registered mainly at home; if aged below 20 years, in $50 \%$ of cases death occurred outdoors, often during physical exercise. In people over 20 years, the role of alcohol among possible trigger factors of SOHCD was substantially increased.

(3) For SOHCD in the age group 1-45 years, the prevalent forensic diagnosis in Russia was "cardiomyopathy." This forensic diagnosis combines specific and nonspecific myocardium changes, in some cases probably developing against a background of fatal arrhythmias or unspecified heart diseases. After 30 years of age, the role of coronary lesions in SOHCD pathogenesis substantially increased.

(4) Based on the revealed frequency, circumstances, and nature of SOHCD, a strategy for early detection of risk groups and sudden cardiac death prophylaxis in young people can be developed.

\section{Conflict of Interests}

The authors declare that there is no conflict of interests regarding the publication of this paper.

\section{References}

[1] R. J. Myerburg and A. Castellanos, "Cardiac arrest and sudden cardiac death," in Heart Disease: A Textbook of Cardiovascular Medicine, E. Braunwald, Ed., pp. 742-779, W.B. Saunders, New York, NY, USA, 1997.

[2] R. F. Gillum, "Sudden coronary death in the United States. 19801985," Circulation, vol. 79, no. 4, pp. 756-765, 1989.

[3] L. G. Escobedo and M. M. Zack, "Comparison of sudden and nonsudden coronary deaths in the United States," Circulation, vol. 93, no. 11, pp. 2033-2036, 1996.

[4] Z.-J. Zheng, J. B. Croft, W. H. Giles, and G. A. Mensah, "Sudden cardiac death in the United States, 1989 to 1998," Circulation, vol. 104, no. 18, pp. 2158-2163, 2001.

[5] C. Basso, E. Carturan, K. Pilichou, S. Rizzo, D. Corrado, and G. Thiene, "Sudden cardiac death with normal heart: molecular autopsy," Cardiovascular Pathology, vol. 19, no. 6, pp. 321-325, 2010.

[6] L. Vertesi, "The paramedic ambulance: a Canadian experience," Canadian Medical Association Journal, vol. 119, no. 1, pp. 25-29, 1978.

[7] J. W. Bachman, G. S. McDonald, and P. C. O'Brien, "A study of out-of-hospital cardiac arrests in northeastern Minnesota," The Journal of the American Medical Association, vol. 256, no. 4, pp. 477-483, 1986.

[8] S. S. Chugh, J. Jui, K. Gunson et al., "Current burden of sudden cardiac death: multiple source surveillance versus retrospective death certificate-based review in a large U.S. community," 
Journal of the American College of Cardiology, vol. 44, no. 6, pp. 1268-1275, 2004.

[9] B. Morentin, B. Aguilera, P. M. Garamendi, and M. P. SuarezMier, "Sudden unexpected non-violent death between 1 and 19 years in north Spain," Archives of Disease in Childhood, vol. 82, no. 6, pp. 456-461, 2000.

[10] G. O. Einarsson, J. Bjornsson, and G. Gunnarsson, "Sudden cardiac death in the young. A 30 year nation-wide study in Iceland," European Heart Journal, vol. 28, Abstract 727, 2007.

[11] R. Margey, A. Roy, S. Tobin et al., "Sudden cardiac death in 14to 35-year olds in Ireland from 2005 to 2007: a retrospective registry," Europace, vol. 13, no. 10, pp. 1411-1418, 2011.

[12] T. J. Bowker, D. A. Wood, M. J. Davies et al., "Sudden, unexpected cardiac or unexplained death in England: a national survey," Monthly Journal of the Association of Physicians, vol. 96, no. 4, pp. 269-279, 2003.

[13] R. R. Liberthson, "Sudden death from cardiac causes in children and young adults," The New England Journal of Medicine, vol. 334, no. 16, pp. 1039-1044, 1996.

[14] C. Wren, J. J. O'Sullivan, and C. Wright, "Sudden death in children and adolescents," Heart, vol. 83, no. 4, pp. 410-413, 2000.

[15] E. Behr, D. A. Wood, M. Wright et al., "Cardiological assessment of first-degree relatives in sudden arrhythmic death syndrome," The Lancet, vol. 362, no. 9394, pp. 1457-1459, 2003.

[16] H. L. Tan, N. Hofman, I. M. van Langen, A. C. van der Wal, and A. A. M. Wilde, "Sudden unexplained death. Heritability and diagnostic yield of cardiological and genetic examination in surviving relatives," Circulation, vol. 112, no. 2, pp. 207-213, 2005.

[17] C. van der Werf, L. Stiekema, H. L. Tan et al., "Low rate of cardiac events in first-degree relatives of diagnosis-negative young sudden unexplained death syndrome victims during follow-up," Heart Rhythm, vol. 11, no. 10, pp. 1728-1732, 2014.

[18] V. Sottas, J.-S. Rougier, F. Jousset et al., "Characterization of 2 genetic variants of Nav 1.5-arginine 689 found in patients with cardiac arrhythmias," Journal of Cardiovascular Electrophysiology, vol. 24, no. 9, pp. 1037-1046, 2013.

[19] D. Corrado, C. Basso, G. Rizzoli, M. Schiavon, and G. Thiene, "Does sports activity enhance the risk of sudden death in adolescents and young adults?" Journal of the American College of Cardiology, vol. 42, no. 11, pp. 1959-1963, 2003.

[20] B. J. Maron, J. J. Doerer, T. S. Haas, D. M. Tierney, and F. O. Mueller, "Profile and frequency of sudden death in 1463 young competitive athletes: from a 25 year U.S. national registry: 19802005," Circulation, vol. 114, no. 18, p. II-830, 2006.

[21] J. Berdowski, M. F. de Beus, M. Blom et al., "Exercise-related out-of-hospital cardiac arrest in the general population: incidence and prognosis," European Heart Journal, vol. 34, no. 47, pp. 3616-3623, 2013.

[22] B. G. Winkel, B. Risgaard, G. Sadjadieh, H. Bundgaard, S. Haunsø, and J. Tfelt-Hansen, "Sudden cardiac death in children (1-18 years): symptoms and causes of death in a nationwide setting," European Heart Journal, vol. 35, no. 13, pp. 868-875, 2014.

[23] M. Kida, T. Kawamura, T. Fukuoka et al., "Out-of-hospital cardiac arrest and survival: an epidemiological analysis of emergency service reports in a large city in Japan," Circulation Journal, vol. 68, no. 7, pp. 603-609, 2004.

[24] M. Kuisma, P. Suominen, and R. Korpela, "Paediatric out-ofhospital cardiac arrests-epidemiology and outcome," Resuscitation, vol. 30, no. 2, pp. 141-150, 1995.
[25] J. A. Drezner, J. S. D. Y. Chun, K. G. Harmon, and L. Derminer, "Survival trends in the United States following exercise-related sudden cardiac arrest in the youth: 2000-2006," Heart Rhythm, vol. 5, no. 6, pp. 794-799, 2008.

[26] I. J. Goldberg, L. Mosca, M. R. Piano, and E. A. Fisher, "Wine and your heart: a science advisory for healthcare professionals from the nutrition committee, council on epidemiology and prevention, and council on cardiovascular nursing of the American Heart Association," Circulation, vol. 103, no. 3, pp. 472-475, 2001.

[27] C. Antzelevich, P. Brugada, J. Brugada et al., The Brugada Syndrome, Future Pbl. Co. Inc., Armonk, NY, USA, 1999.

[28] A. J. Fuenmayor and A. M. Fuenmayor, "Cardiac arrest following holiday heart syndrome," International Journal of Cardiology, vol. 59, no. 1, pp. 101-103, 1997.

[29] K.-J. Bär, M. K. Boettger, M. Koschke et al., "Increased QT interval variability index in acute alcohol withdrawal," Drug and Alcohol Dependence, vol. 89, no. 2-3, pp. 259-266, 2007. 


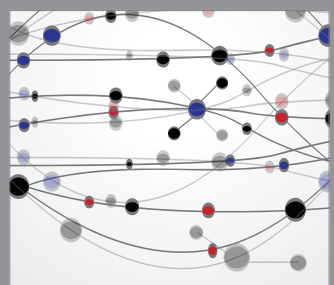

The Scientific World Journal
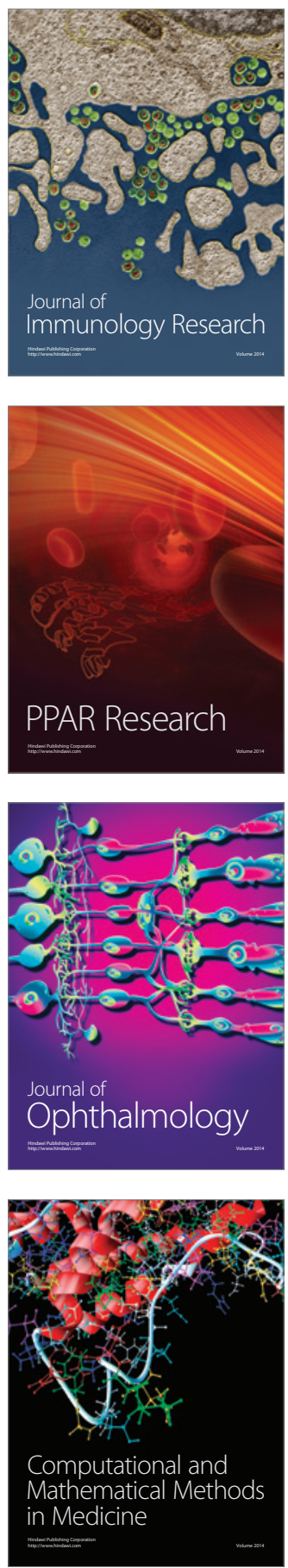

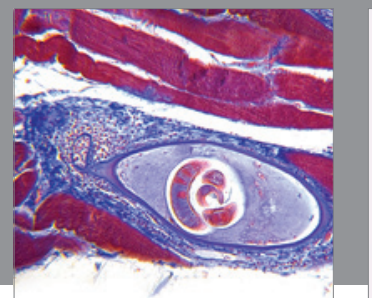

Gastroenterology

Research and Practice
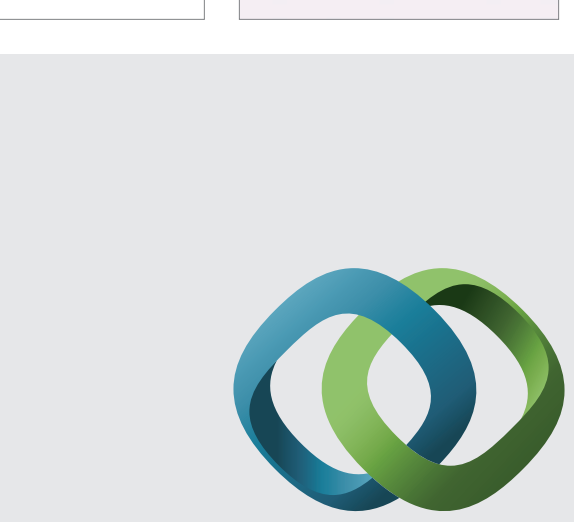

\section{Hindawi}

Submit your manuscripts at

http://www.hindawi.com
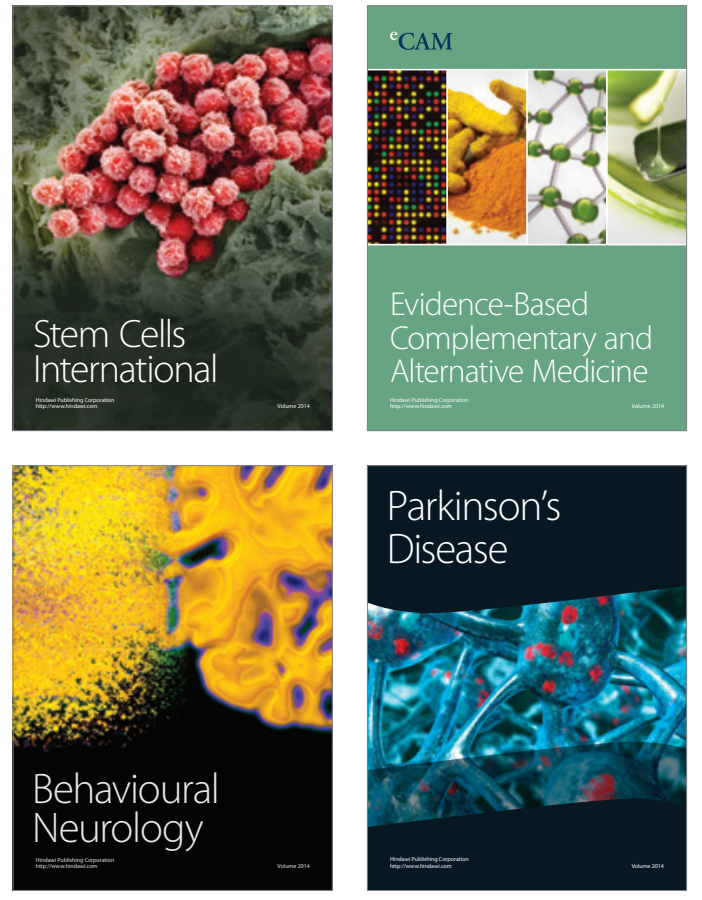
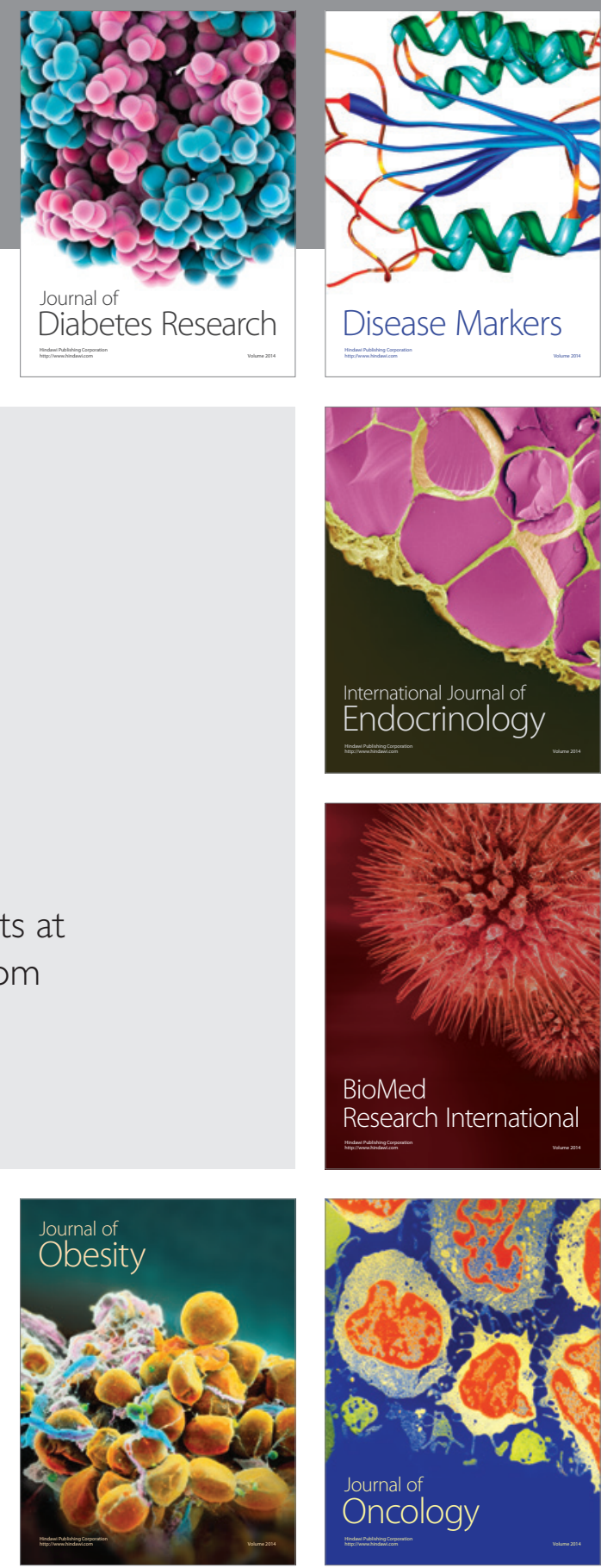

Disease Markers
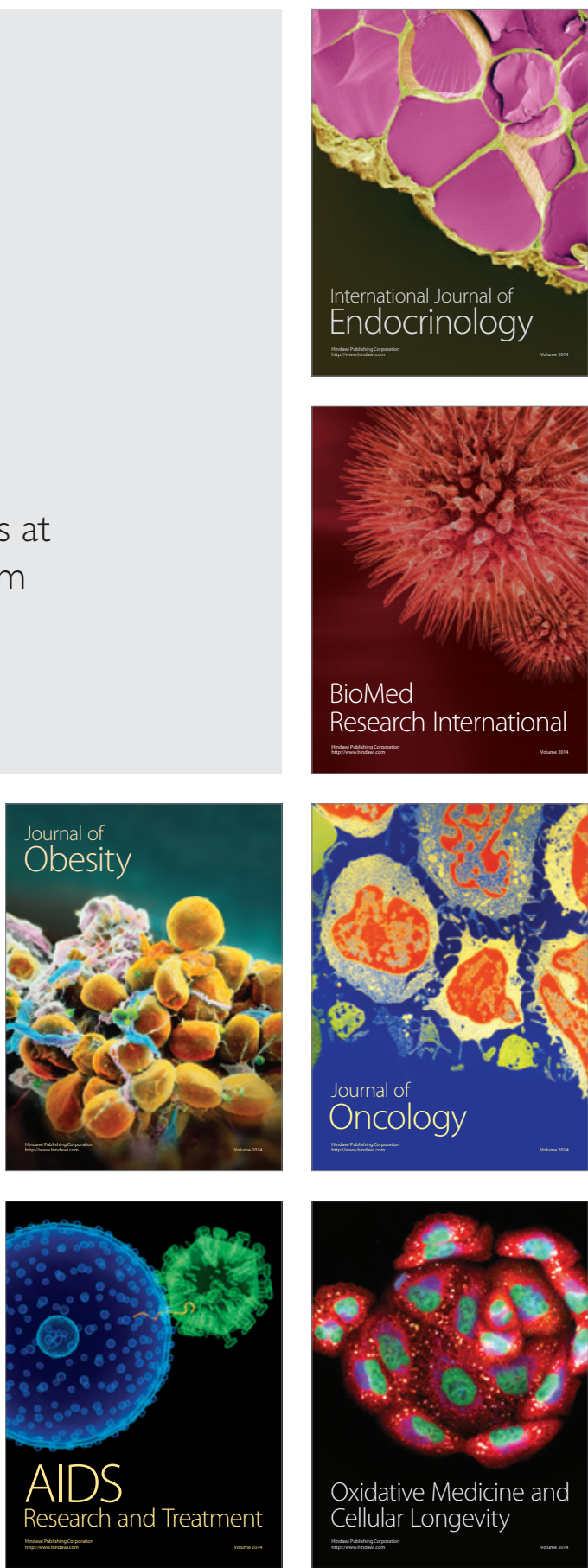